\title{
Capture of complete ciliate chromosomes in single sequencing reads reveals widespread chromosome isoforms
}

\author{
Kelsi A. Lindblad ${ }^{1,2}$, Jananan S. Pathmanathan', Sandrine Moreira', John R. Bracht ${ }^{3}$, Robert P. Sebra ${ }^{4}$, \\ Elizabeth R. Hutton ${ }^{2,5}$ and Laura F. Landweber ${ }^{1 *}$
}

\begin{abstract}
Background: Whole-genome shotgun sequencing, which stitches together millions of short sequencing reads into a single genome, ushered in the era of modern genomics and led to a rapid expansion of the number of genome sequences available. Nevertheless, assembly of short reads remains difficult, resulting in fragmented genome sequences. Ultimately, only a sequencing technology capable of capturing complete chromosomes in a single run could resolve all ambiguities. Even "third generation" sequencing technologies produce reads far shorter than most eukaryotic chromosomes. However, the ciliate Oxytricha trifallax has a somatic genome with thousands of chromosomes averaging only $3.2 \mathrm{kbp}$, making it an ideal candidate for exploring the benefits of sequencing whole chromosomes without assembly.
\end{abstract}

Results: We used single-molecule real-time sequencing to capture thousands of complete chromosomes in single reads and to update the published Oxytricha trifallax JRB310 genome assembly. In this version, over 50\% of the completed chromosomes with two telomeres derive from single reads. The improved assembly includes over 12,000 new chromosome isoforms, and demonstrates that somatic chromosomes derive from variable rearrangements between somatic segments encoded up to 191,000 base pairs away. However, while long reads reduce the need for assembly, a hybrid approach that supplements long-read sequencing with short reads for error correction produced the most complete and accurate assembly, overall.

Conclusions: This assembly provides the first example of complete eukaryotic chromosomes captured by single sequencing reads and demonstrates that traditional approaches to genome assembly can mask considerable structural variation.

Keywords: Ciliate, Oxytricha, PacBio, SMRT sequencing, Genome assembly, Alternative fragmentation

\section{Background}

Whole-genome shotgun sequencing, first pioneered in eukaryotes during the human genome project, has become such common practice that over 38,000 genome assemblies are available from NCBI today [1]. Despite its ubiquity, genome assembly is still a challenge, requiring the computation of overlaps among millions of short reads. In particular, the use of short reads makes it difficult to place repetitive elements, resolve the length of microsatellite

\footnotetext{
* Correspondence: Laura.Landweber@columbia.edu

'Departments of Biochemistry \& Molecular Biophysics and Biological

Sciences, Columbia University, New York, NY 10032, USA

Full list of author information is available at the end of the article
}

repeats, or capture haplotypes over large genomic regions. Traditional whole-genome shotgun sequencing leaves much to be desired for non-model genomes that exhibit either long repeats or high polymorphism rates that fall outside the assumptions of most assembly programs, such as plant genomes that contain high levels of repetitive elements and high ploidy [2,3], or genomes with large stretches of similarity that result from whole-genome duplications, such as the ciliate Paramecium [4]. Despite improvements in assembly algorithms, the best way to completely overcome these issues would ultimately be to use a sequencing method capable of accurately reading the sequence of each chromosome in full. Although current

(c) The Author(s). 2019 Open Access This article is distributed under the terms of the Creative Commons Attribution 4.0 International License (http://creativecommons.org/licenses/by/4.0/), which permits unrestricted use, distribution, and reproduction in any medium, provided you give appropriate credit to the original author(s) and the source, provide a link to the Creative Commons license, and indicate if changes were made. The Creative Commons Public Domain Dedication waiver (http://creativecommons.org/publicdomain/zero/1.0/) applies to the data made available in this article, unless otherwise stated. 
sequencing technologies fall far short of this mark, read lengths have increased substantially. Pacific Biosciences' single-molecule real-time (SMRT) sequencing platform achieves read lengths as high as 50,000 base pairs [5], while reads over 200,000 base pairs long have been reported from Oxford Nanopore's MinION [6]. The higher resolution provided by these long reads has made it possible to produce high-quality reference sequences that capture structural variation that short-read sequencing cannot resolve $[7,8]$ and even automate the completion of microbial genomes [9].

While it is not yet possible to produce reads long enough to capture most eukaryotic chromosomes, Oxytricha trifallax's tiny "nanochromosomes" fall well within the range of recent long-read sequencing technologies and, themselves, offer powerful models for studying eukaryotic chromosome biology [10, 11]. Like all ciliates, Oxytricha has two nuclear genomes, a transcriptionally silent germline and a compressed somatic genome used for most of the cell's transcription. The germline genome has a complex architecture containing $>225,000$ short genic sequences (macronuclear destined sequences, MDSs) that assemble during development to form the somatic genome. In addition, approximately $22 \%$ of MDSs are present in a permuted order or inverse orientation in the germline, and require descrambling during formation of the somatic chromosomes, together with removal of thousands of noncoding sequences (internally eliminated sequences, IESs) that interrupt MDSs [12]. While the germline genome contains hundreds of long chromosomes, the somatic genome is highly fragmented with $\sim 20,000$ different chromosomes that average just $3.2 \mathrm{~kb}$ in length $[13,14]$, possess very few well-positioned nucleosomes [10], and derive from a copy of the germline through an elaborate process of RNA-guided genome rearrangement that eliminates 90 95\% of the germline sequence, including all IESs, stitches together the remaining germline segments in the correct order $[15,16]$, and adds telomeres to chromosome ends (reviewed in Yerlici and Landweber [17]).

In addition to small chromosome size, Oxytricha's somatic genome displays several features that complicate traditional genome assembly. Approximately $25 \%$ of chromosomes contain one or more internal sites used for telomere addition, which terminates the chromosome. The same proportion of chromosomes use alternative recombination between germline segments. The use of internal telomere addition sites and alternative chromosome fragmentation produces a family of chromosome isoforms that contain only part of another chromosome's sequence [14]. Furthermore, somatic chromosomes exhibit copy number variation that can range over orders of magnitude, which is well outside the assumptions of most assembly programs and sequencing techniques.
However, while long-read sequencing has the potential to solve many issues associated with the assembly of Oxytricha's macronuclear genome, it also has a major drawback: SMRT sequencing and other long-read technologies produce reads with a much higher error rate than those produced by shortread sequencers. Raw PacBio reads may have up to a $13 \%$ error rate, compared to a $\sim 0.5 \%$ error rate for Illumina [18]. The raw reads therefore require an additional pre-processing "error correction" step prior to assembly. Traditionally, this has been accomplished by aligning short reads to errorcontaining long reads and using a consensus call method to infer the correct sequence of the long read. The advent of pipelines like PBCR that produce corrected long reads by aligning raw long reads to long reads [5], may eliminate the need for pre-processing correction with short read sequencing but they require much greater PacBio coverage. After self-correction, PacBio reads still exhibit a basal error rate of $\sim 2-3 \%$ [19], compatible with modern assemblers, but the resulting assembly needs to be further improved with postassembly correction by short-reads.

In 2013, our lab published a high-quality assembly of Oxytricha's somatic genome using a combination of Sanger, 454 and Illumina data. Here we present an updated version incorporating SMRT sequencing. The improved assembly includes over 13,000 complete chromosomes captured in single reads, entirely without assembly. We find that long reads are ideal for capturing the large number of structural variants in the Oxytricha somatic genome and discuss the relative merits of different sequencing strategies for producing the highest-quality assembly for an extensively fragmented genome.

\section{Results}

\section{Over half the Oxytricha somatic genome can be} completely sequenced without assembly

We isolated Oxytricha trifallax strain JRB310 somatic, macronuclear DNA for SMRT sequencing, combining a pilot sequencing run using the P2 chemistry with a second, full run using P3 chemistry, for a total of 10 SMRT cells and 264x genome coverage (Table 1). After filtering

Table 1 SMRT sequencing of the Oxytricha somatic genome

\begin{tabular}{|c|c|c|c|c|}
\hline & P2 Chemistry & P3 Chemistry & Combined & Self-corrected \\
\hline $\begin{array}{l}\text { Number of } \\
\text { Flow Cells }\end{array}$ & 2 & 8 & 10 & $-/-$ \\
\hline Total Subreads & 584,388 & $4,622,662$ & $5,207,050$ & $1,637,578$ \\
\hline $\begin{array}{l}\text { Total Sequence } \\
\text { (GB) }\end{array}$ & 1.37 & 11.90 & 13.27 & 3.5 \\
\hline $\begin{array}{l}\text { Mean Read } \\
\text { Length (bp) }\end{array}$ & 2350 & 2575 & 2545 & 2152 \\
\hline $\begin{array}{l}\text { Max Read } \\
\text { Length (bp) }\end{array}$ & 32,258 & 42,863 & 42,863 & 13,629 \\
\hline $\begin{array}{l}\text { Genome } \\
\text { Coverage }^{\text {a }}\end{array}$ & $26 x$ & $238 x$ & $264 x$ & $70 x$ \\
\hline
\end{tabular}

${ }^{\mathrm{a}}$ Based on a genome size of $50 \mathrm{MB}$ 
and self-correction, we recovered 599,310 reads. As expected, the distribution of sequencing read lengths closely matches the length distribution of Oxytricha somatic chromosomes (Fig. 1), and 324,445 corrected subreads contained telomeric sequences on both ends, indicating that they are complete chromosomes. These reads with two telomeres represent 11,378 distinct chromosomes or $51 \%$ of the contigs in the published assembly; thus, over half of the genome can be completely sequenced without assembly. We used the Celera Assembler to assemble the corrected reads that lacked telomeric sequences on both ends into contigs and combined these contigs with the single-read chromosomes to produce a long-read-only assembly (Pure PacBio Assembly) (Table 2). Although this assembly contains over 9000 more contigs than the previously published assembly, the majority of the new additions are alternatively fragmented isoforms of previously sequenced chromosomes (Fig. 2 and see "Long-read sequencing discovers novel chromosome isoforms"). While
SMRT sequencing provided good coverage of chromosomes around the somatic genome's mean 3.2kbp length, it was unable to capture most of the shortest chromosomes, largely because short reads $(<300 \mathrm{bp})$ were filtered out at several points during the data cleaning process. The shortest gene-containing two-telomere chromosome in our assembly was $314 \mathrm{bp}$, compared to $502 \mathrm{bp}$ in the published assembly. Meanwhile, the longest chromosome captured by a single read was $13,906 \mathrm{bp}$, which encodes three genes including a Serine/Threonine kinase. Overall, $13 \%$ of contigs $\geq 10,000 \mathrm{bp}$ were present in the long read data, compared to $63 \%$ of contigs between $1000 \mathrm{bp}$ and $10,000 \mathrm{bp}$. This indicates that SMRT sequencing was able to capture long chromosomes in addition to short ones.

To produce a final assembly that combines the strengths of the short read assembly with long read data, we combined our pure long read (PacBio) assembly with high-confidence contigs from the published assembly to create a hybrid assembly containing all high-confidence

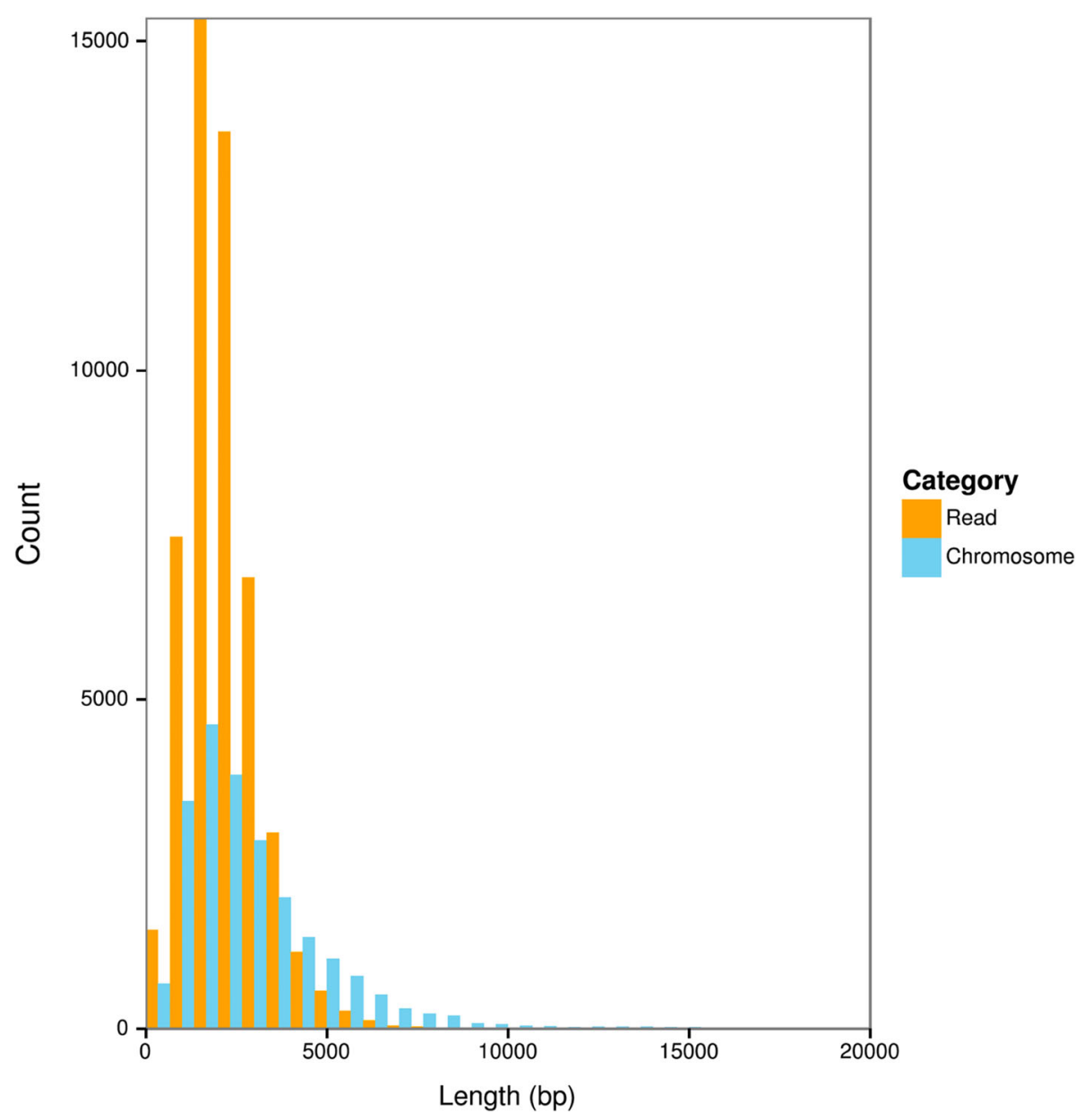

Fig. 1 SMRT sequencing reads are long enough to capture complete Oxytricha chromosomes. The length distribution of corrected SMRT subreads is similar to the length distribution of Oxytricha chromosomes. To improve readability, a random subsample of 50,000 SMRT subreads is shown, and the twelve chromosomes longer than 20,000 bp (from 22,000 bp to 66,000 bp) have been omitted from the plot 
Table 2 Assembly statistics for long- and short-read genome assemblies

\begin{tabular}{llll}
\hline & $\begin{array}{l}\text { Published } \\
\text { Assembly [14] }\end{array}$ & $\begin{array}{l}\text { Pure PacBio } \\
\text { Assembly }\end{array}$ & $\begin{array}{l}\text { Hybrid } \\
\text { Assembly }\end{array}$ \\
\hline Assembly Size & $64 \mathrm{MB}$ & $87 \mathrm{MB}$ & $108 \mathrm{MB}$ \\
$\begin{array}{l}\text { Total Contigs } \\
\begin{array}{l}\text { Unique Predicted } \\
\text { Proteins a }\end{array}\end{array}$ & 22,450 & 31,664 & 38,668 \\
$\begin{array}{l}\text { \% Identified } \\
\text { Busco genes }\end{array}$ & 24,963 & 16,251 & 27,528 \\
$\begin{array}{l}\text { \% Illumina read } \\
\text { mapping }\end{array}$ & $95 \%$ & $58 \%$ & $85 \%$ \\
\hline
\end{tabular}

a Based on a $90 \%$ similarity cut off

chromosome isoforms identified by either approach. The hybrid assembly was scrutinized and polished by correcting remaining PacBio sequencing errors with Illumina reads. Of the 38,668 contigs in the final assembly, alternative fragmentation detection revealed 18,617 distinct chromosomes, with 5226 possessing at least one isoform. Note that this number of distinct chromosomes is approximately 2000 more than previously reported in Swart et al. [14]. The previously published genome assembly had been judged largely complete based on its complement of tRNA genes and overlap with the CEG database of core eukaryotic proteins [14]. Here, we measured the completeness of the published and hybrid Assembly using BUSCO [20, 21], both assemblies showed a similar completeness score of $85 \%$. While $100 \%$ would be the ideal level of genome completeness expected from BUSCO, this is just one metric for assessing the quality of an assembly. Our lab previously published a study [22] that assembled the somatic genome of six ciliates and assessed completeness using the representation of core eukaryotic genes (CEGs). When we rechecked the completeness of these genomes using BUSCO it produced a range of scores from 70 to $85 \%$. Furthermore, in Chen et al. 2018 [23] the authors used BUSCO to evaluate the completeness of the Euplotes vannus genome, as well as the Oxytricha and Tetrahymena genomes, and observed a similar trend. Moreover, the complement of unique predicted proteins is much higher for the hybrid assembly because we used RNA-seq data from vegetatively-growing, starved, and encysted Oxytricha cells for gene prediction with Augustus. The domain analysis of these proteins shows that the hybrid assembly contain only 94 more protein domains that were not identified in the previous assembly. This suggests that, rather than having missed large

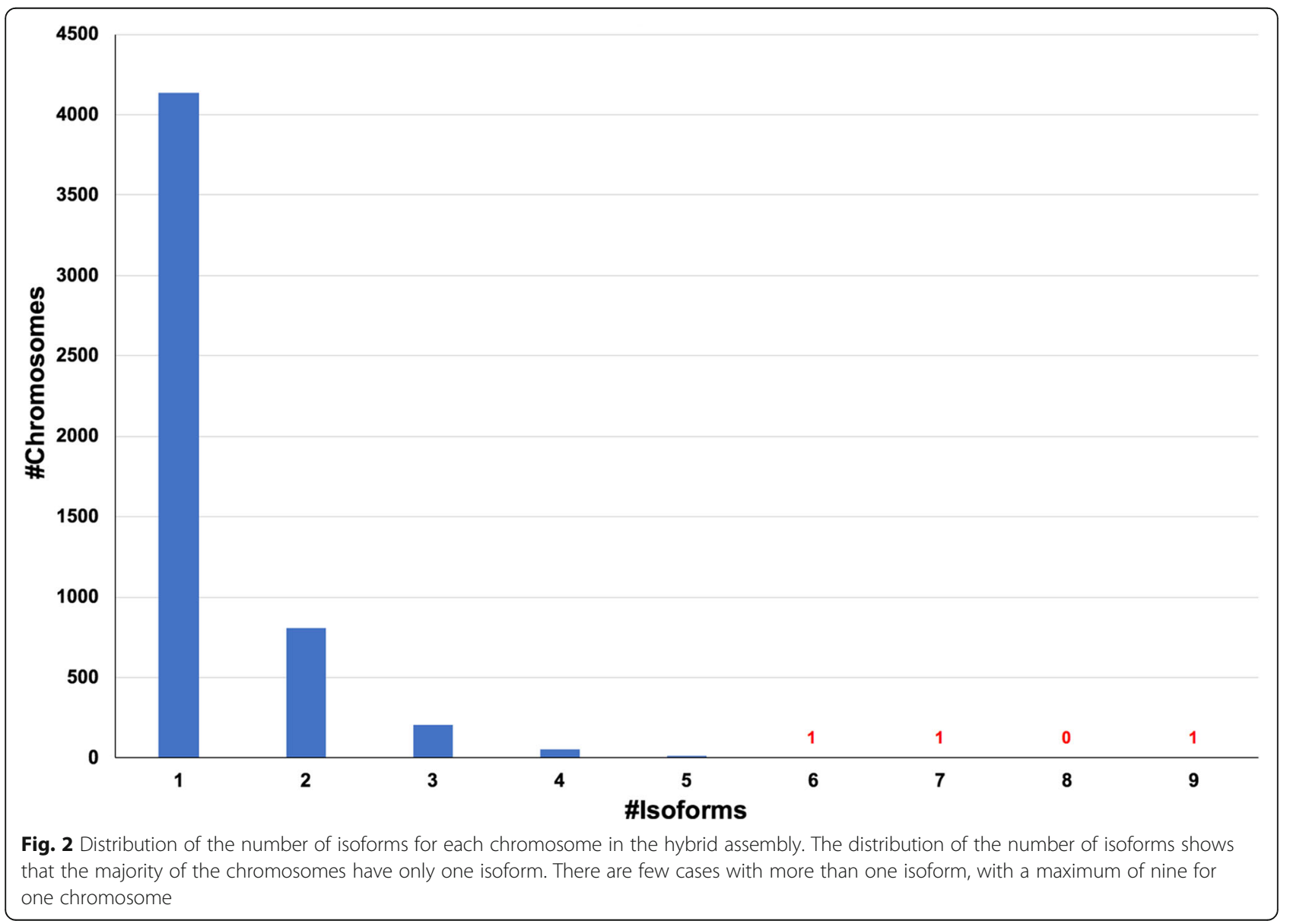


numbers of functional proteins in the previous assembly, the larger proteome size in the hybrid assembly is mostly accounted for by the presence of variants of existing proteins. Also, while approximately 13,500 new chromosome variants were identified in the long read data, only two entirely new, incomplete chromosomes were discovered. This suggests that the hybrid assembly is virtually complete.

\section{Long-read sequencing discovers novel chromosome isoforms}

Oxytricha's somatic chromosome isoforms are often masked by genome assembly pipelines that merge short chromosomes into larger ones with the same sequence. Previous estimates of the level of alternative fragmentation in Oxytricha were based either on PCR examination of individual loci [24] or on the inference of telomere addition sites by identifying pileups of telomerecontaining reads [14]. SMRT sequencing captures these variants in their entirety. Our genome-wide analysis of alternative fragmentation sites identified 25,312 distinct chromosome variants, with 5226 of the 18,617 (28\%) detected chromosomes demonstrating at least one alternative fragmentation site (Fig. 2). The functional analysis of the proteins encoded by these chromosomes with isoforms suggests that they are mostly involved in cellular processes and signaling functions (Fig. 3). They are enriched in three KOG functional categories: "T" (Signal transduction mechanisms), "O" (Posttranslational modification, protein turnover, chaperones) and "U" (Intracellular trafficking, secretion, and vesicular transport). Curiously, chromosomes that lack isoforms in our study display an excess of predicted proteins with unknown functions.

Mapping telomere-containing Sanger sequencing reads predicted 6695 isoforms [14], 76\% of which are also found among the isoforms in the long read assembly. This indicates that SMRT sequencing captures the same kind of isoforms, but notably it finds more of them.

Furthermore, the ability of long read sequencing to retrieve complete sequences of the isoforms, not just their lengths, allows us to examine alternative fragmentation on a genome-wide scale. Previous studies have inferred that multiple germline loci may contribute to families of alternative fragmentation isoforms [24, 25]. With the current data provided by long-read sequencing, we find that some isoforms may derive from mixing and matching between these different loci, rather than from processing each locus separately. Figure 4 shows one of the chromosomes with the most fragmentation isoforms in our dataset, Contig14329.0, that has nine isoforms. Of these, four incorporate sequence from two separate germline loci, suggesting that alternative fragmentation and assembly can recombine segments from multiple loci, which would require inter-chromosomal

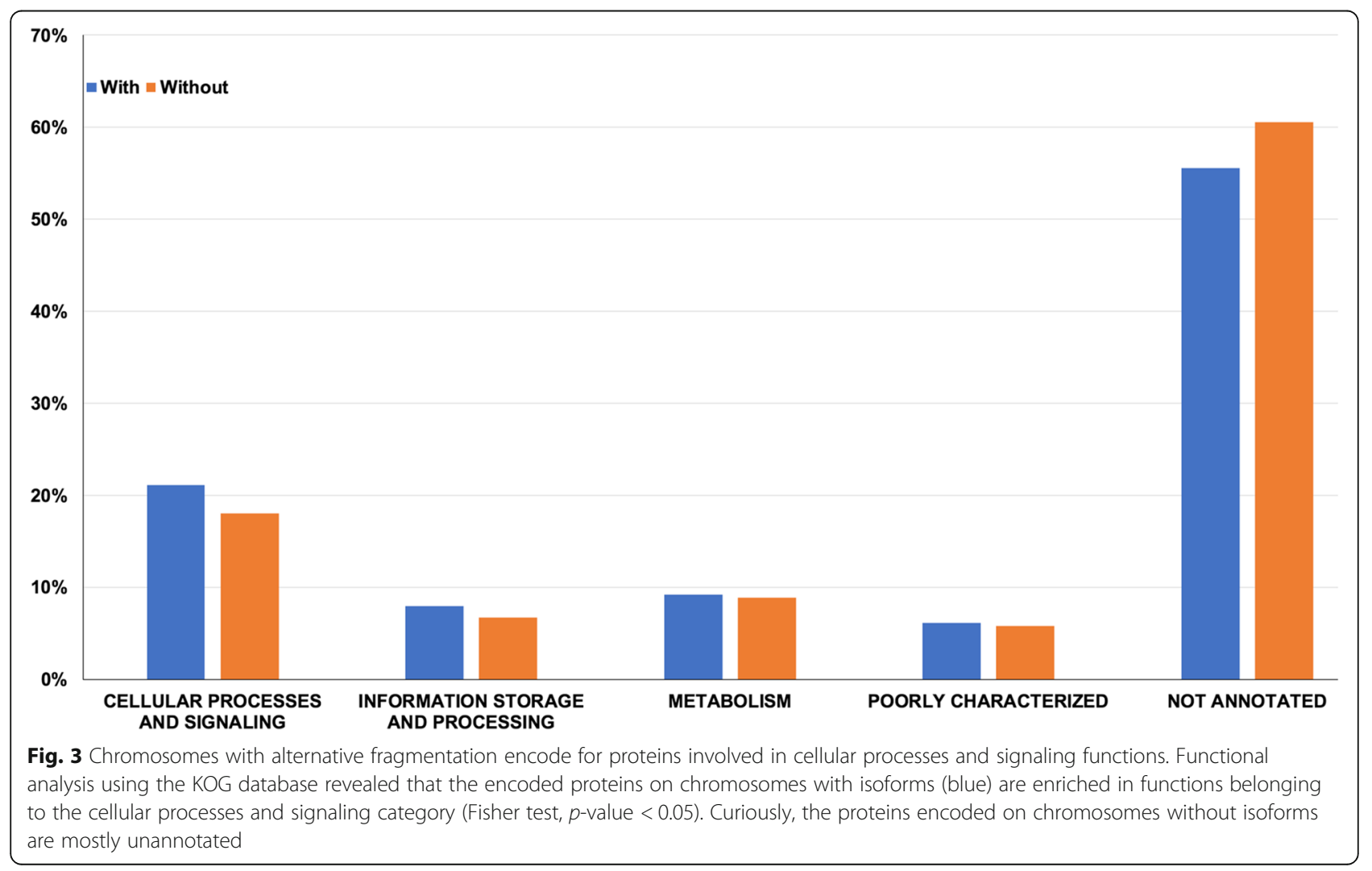




\section{Somatic Isoforms}

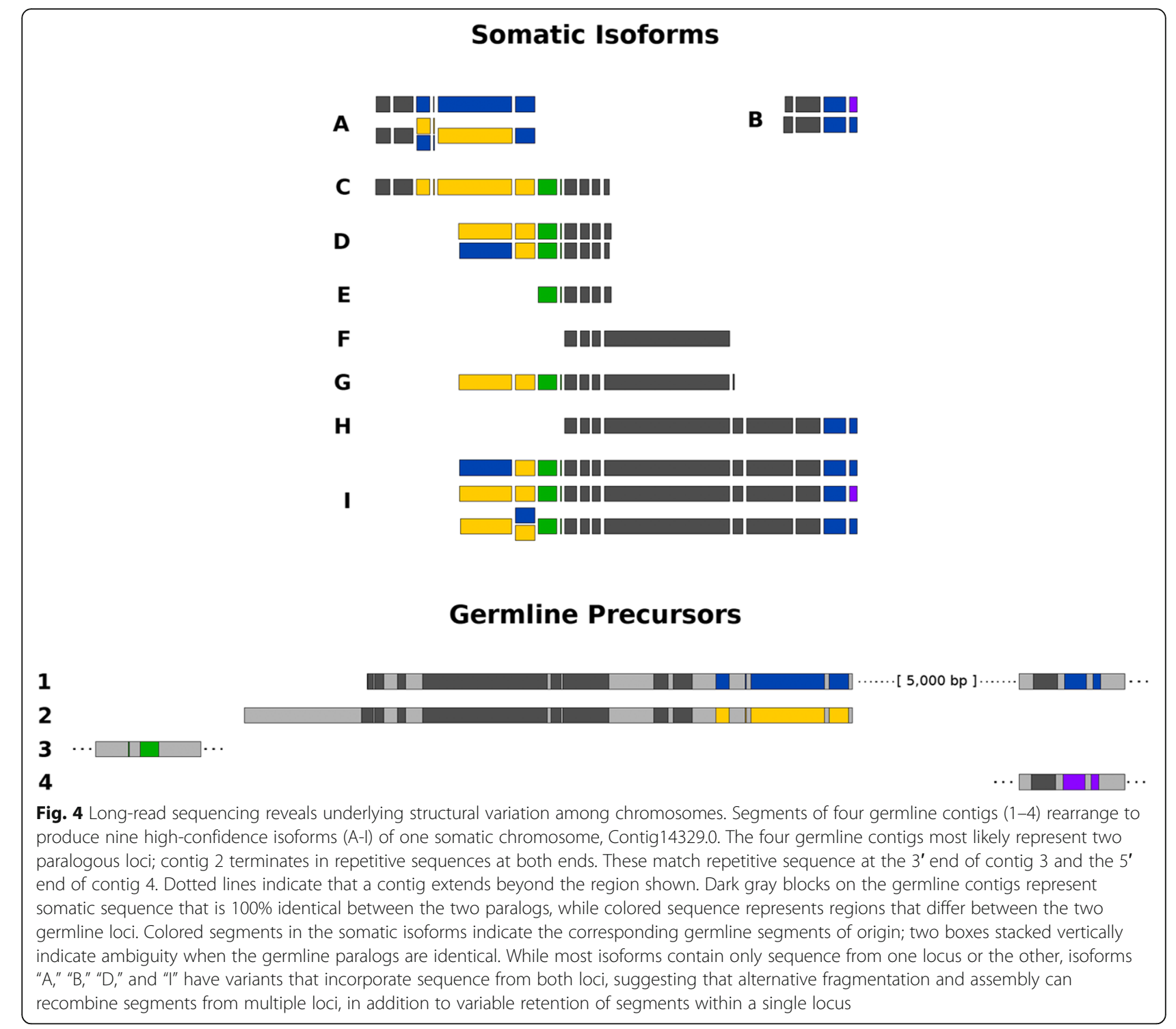

recombination. The other five isoforms include segments from only one locus or the other. To produce the full complement of isoforms for this chromosome the cell must therefore undertake variable processing within a single locus, as well as combine sequences from multiple loci. Several of the alternatively fragmented isoforms also contain segments from just one locus or the other, suggesting that an unknown mechanism might regulate which isoforms a locus produces. We find that variable processing is widespread, with 2522 out of 5226 (48\%) alternatively fragmented chromosomes deriving from two or more paralogous germline loci. Moreover, it will be illuminating to mine the data for evidence of interallelic rearrangements in Oxytricha's somatic genome. However, we found that the current data and methods were insufficient to phase each chromosome from the hybrid assembly to produce a high quality haploid version of the genome assembly.
Hybrid error correction produces the most complete somatic genome assembly

To determine whether pure long-read sequencing produces an assembly of similar quality to a hybrid strategy that uses short reads to correct PacBio reads, we subsampled our long read data and assessed the completeness of assemblies produced using the two correction methods. Overall, hybrid error correction outperforms long-read-only error correction at all sequencing depths (Fig. 5), and while the number of contigs recovered by hybrid error correction begins to saturate with eight flow cells' worth of data, the steep slope of the long-read-only curve suggests that considerably more sequencing depth would be necessary to correct all chromosomes using only long reads.

The heterogeneous copy number of chromosomes in Oxytricha's somatic genome may be the root cause for 


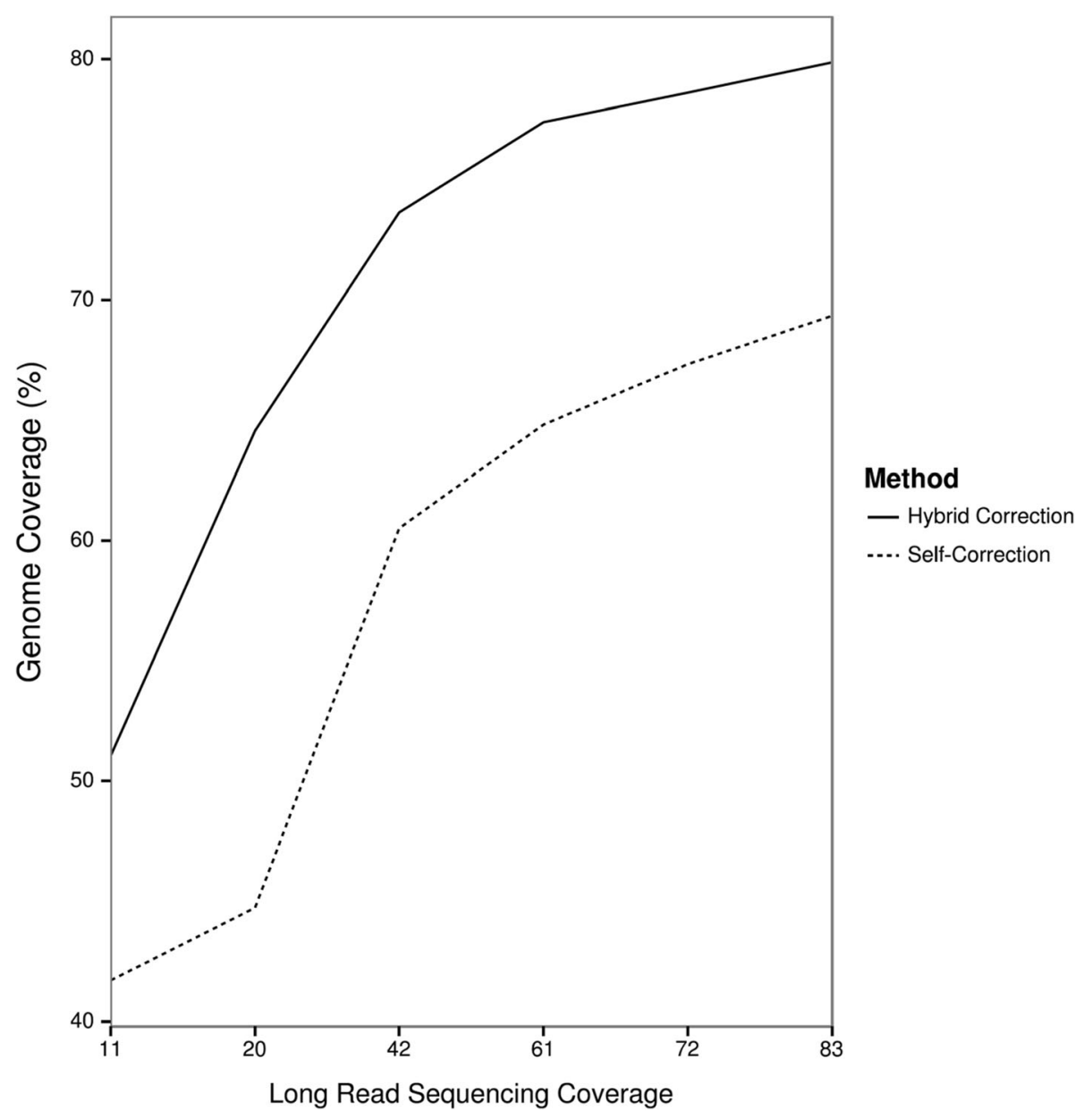

Fig. 5 Hybrid error correction outperforms long read self-correction. With 50x coverage of short-read data, hybrid error correction produces a more complete assembly than self-correction, even at twice the minimum recommended long-read coverage

the inadequacy of long-read error correction. While the average somatic chromosome copy number is approximately 2000n [13], some chromosomes can be amplified to over 200,000 copies. This reduces the effective coverage for low-copy number chromosomes, as more abundant chromosomes absorb a disproportionate amount of sequencing depth. Both the hybrid-corrected and selfcorrected genome assemblies were biased towards chromosomes with significantly greater copy number than average read coverage, even when all flow cells were incorporated (Welch's one-sided $t$-test, $t=4.1652, p=$ $1.559 \mathrm{e}-05$ for hybrid correction, $t=4.7637, \mathrm{p}=1.559 \mathrm{e}-05$ for self-correction). However, hybrid error correction resulted in a steeper decline in mean chromosome copy number across the genome as sequencing depth increased, compared to self-correction ( 79 fewer for the hybrid error correction, 13 fewer for self correction). This indicates that the hybrid error correction incorporated more lowabundance chromosomes as the amount of long read data increased, relative to the self-correction method. While we recovered 135x coverage of corrected sequence from strictly long reads, this derived from only hundreds of thousands of PacBio reads, each an individually sampled molecule, compared with tens of millions of short-read Illumina sequences. The increased depth that can be achieved with short reads is thus more important to the completeness of the final genome assembly than the increased resolution provided by long-read sequencing.

\section{Discussion}

As long-read sequencing technology improves, it may eventually be possible to sequence complete chromosomes of most organisms in a single contiguous read. For now, Oxytricha's highly fragmented genome provides the first opportunity for genome sequencing without assembly. This approach permitted the discovery of structural chromosome isoforms that were masked by traditional assembly pipelines. Long-read sequencing dramatically increased the number of alternative isoforms that we could identify. Furthermore, we conclude 
that many of these isoforms may derive from recombination among multiple germline loci, in addition to variable processing within a locus. Where allelic variation is present, this implies that genome rearrangement may occur between-as well as within-germline chromosomes. The observation that some isoforms derive exclusively from one locus or the other also raises the question of what regulates this selection and what determines the range of isoforms produced. The finding that piRNAs can strongly influence chromosome fragmentation patterns and lead to alternatively processed chromosomes [26] suggests that the piRNA pathway is involved $[27,28]$ in this process.

The long read sequencing in this study permitted a higher quality examination of closely-related chromosome isoforms than the previously published assembly. However, for de novo genome assembly, the variation in chromosome copy number in Oxytricha makes the approach less economical than short-read sequencing. Alternatively, for species that possess highly fragmented genomes and genesized chromosomes with variable chromosome copy number, the challenge of genome assembly is similar to transcriptome assembly. As such, the PacBio isoform sequencing (Iso-Seq) pipeline for transcriptome assembly could also be modified for genome sequencing, just as it is capable of capturing complete transcripts without assembly and identifying novel genes and isoforms produced via alternative splicing [29-32]. Overall, we recommend that future studies perform an initial assembly based on short read data and use long reads to investigate structural variants, the area where we reaped the most benefit for this genome.

\section{Conclusions}

The combination of high coverage long and short reads permits the most complete assembly of a ciliate genome, together with the discovery of novel structural variants. The improved Oxytricha trifallax macronuclear genome assembly presented here will allow further investigation of chromosome rearrangements in this species and lineage.

\section{Methods}

\section{Cell growth and culture}

Cell growth, harvest, and nuclei isolation of Oxytricha trifallax strain JRB310 were carried out as described in [12], with the exception that the pellet was collected after the initial centrifugation step rather than from the $10 \%$ gradient fraction to isolate macronuclei rather than micronuclei.

\section{Library preparation and sequencing}

Library preparation and sequencing were per the manufacturer's instructions for P5-C3 and P6-C4 sequencing enzyme and chemistry, as previously described [12]. Aliquots of $5 \mu \mathrm{g}$ of extracted high-quality genomic DNA were enriched for MAC DNA and verified using Qubit analysis. DNA was quantified and diluted to $150 \mu \mathrm{L}$ in Qiagen elution buffer $(33 \mu \mathrm{g} / \mu \mathrm{L})$. The sample was pipetted into the top chamber of a Covaris G-tube spin column, gently sheared $60 \mathrm{~s}, 4500 \mathrm{rpm}$ in an Eppendorf 5424 bench top centrifuge, followed by $0.45 \mathrm{X}$ AMPure $\mathrm{XP}$ purification. $\sim 1.2 \mu \mathrm{g}$ of this sample was used in library preparation exactly as described in [12].

After library preparation, samples were validated as $5 \mathrm{~kb}$ via an Agilent DNA 12000 gel chip. Blue Pippin $0.75 \%$ agarose cassettes (Sage Science) were used to prepare a MAC-enriched library (5000 bp - 50,000 bp). In 2014 we sequenced two SMRT Cells as a proof of concept. For these initial SMRT Cells the polymerasetemplate complex was bound to the P5 enzyme using a ratio of 10:1 polymerase to SMRTbell at $0.5 \mathrm{nM}, 4 \mathrm{~h}$, $30{ }^{\circ} \mathrm{C}$, then incubated at $4{ }^{\circ} \mathrm{C}$ prior to magbead loading and sequencing with the $\mathrm{C} 3$ chemistry. In 2015 we sequenced 8 additional SMRT Cells to have enough material for long read self-correction. For these additional SMRT Cells the complex was bound to the P6 enzyme and sequenced using the $\mathrm{C} 4$ chemistry. The magnetic bead-loading step was conducted at $4{ }^{\circ} \mathrm{C}$ for $60 \mathrm{~min}$. The magbead-loaded, polymerase-bound SMRTbell libraries were placed onto the RSII machine at a sequencing concentration of 100 to $110 \mathrm{pM}$ and sequenced across two SMRT Cells using P5-C3 and 8 additional SMRT Cells using P6-C4 chemistry.

\section{Genome assembly}

We used Pacific Bioscience's SMRT Pipe 2.3.0 [33] to quality trim and to filter raw SMRT sequencing reads, using default parameters but enabling the artifact filter (parameter value -1000) in order to remove chimeric reads. Reads that passed the filter were self-corrected using PBcR (default parameters) [5].

Error correction deleted the telomeres from most reads, so we gathered all raw reads that had at least one telomere, based on matching to the regular expression [TG]"TTTTGGGGTTTT, [TG]"GGGGTTTT GGGG, [AC]"AAAACCCCAAAA, or [AC]"CCCCAA AACCCC with an edit distance of two. The first and last $1000 \mathrm{bp}$ of these reads were corrected using ECTools (default parameters) [34, 35] and a 50x coverage subset of Illumina reads from the previously published Oxytricha somatic assembly [14]. Chromosome ends corrected in this manner were aligned to the PBcR-corrected read and the missing bases filled in from the ECTools corrected read.

Some corrected reads were chimeras of multiple chromosomes, characterized by embedded telomeric sequences, or sequencing artifacts composed almost exclusively of 
homopolymer runs. As a result, we filtered out all corrected reads containing a homopolymer run of $>10 \mathrm{bp}$ or a non-terminal telomeric sequence (matching the regular expression [AC]*(CCAAAACCCCAAAA) or (GGTTTTGGGGTTTT)[TG] with an edit distance of one or [AC] "CCCAAAACCCCGGGGTTTTGGG[TG*] or [TG]"GGGTTTTGGGGCCCCAAAACCC[AC*] with an edit distance of three).

After filtering, all reads with telomeric sequences on both ends were considered complete chromosomes and retained, while reads with one or fewer telomeres were assembled using Celera Assembler 8.3rc [5]. We combined the assembled contigs with the two-telomere reads and clustered the resulting sequences at a $90 \%$ identity threshold using VSEARCH [36] and took the centroid contig for each of the resulting clusters to produce a final set of unique chromosomes.

We removed duplicated sequences with BBTools dedupe.sh script [37]. We polished our assembly by recursively applying Pilon [38], an error correction tool that uses Illumina reads to correct PacBio sequencing errors.

We determined alternative fragmentation isoforms by extracting all two-telomere single reads and contigs from our data and masking the telomeres according to the procedure described in [14]. We then used BWA MEM [39] to map the masked reads against the subset of unique chromosomes in our assembly. We grouped all reads with both start and end positions within $50 \mathrm{bp}$ of one another into distinct isoforms and clustered all reads assigned to each isoform at a $97 \%$ similarity threshold. We added the consensus sequence of each cluster comprising at least two contigs to the assembly.

To finalize the assembly, we added contigs that were captured in the published Oxytricha assembly but not in our long-read assembly. These included two-telomere contigs shorter than $600 \mathrm{bp}$ long and contigs either without an analog in the long-read data, or where the longest isoform in the long-read assembly was at least $75 \mathrm{bp}$ shorter than the version in the published assembly. In cases where the published contig was longer and the long-read version had both telomeres, we considered the long-read form an alternative fragmentation isoform and retained it in addition to adding the longer published contig. If the long-read form had fewer than two telomeres, it was discarded instead. Finally, we removed contigs where at least $50 \%$ of the contig sequence was covered by a known germline repetitive element or satellite repeat. We also removed as likely contaminants any contigs without any telomeres and which were less than $20 \%$ covered in the germline genome.

\section{Analysis of alternative chromosome fragmentation}

To compare the alternative fragmentation isoforms found by SMRT sequencing with those predicted by older sequencing technologies, we masked all two-telomere corrected reads as described above and mapped them against the published somatic genome assembly [14]. We then grouped reads into distinct isoforms as described above, choosing only the longest hit for each read. In addition, because a size selection step was used in the Sanger sequencing that produced the original predicted isoforms, we filtered the resulting isoforms to include only those less than $6000 \mathrm{bp}$ long. To determine whether an isoform found by one method was also discovered by the other, we used BEDTools 2.25.0 intersect [40] with the options -F 90 -f 90 to count only isoforms that were at least $90 \%$ covered in both assemblies.

To analyze how somatic isoforms relate to their germline loci, we selected all isoforms supported by at least two corrected reads and aligned them to the germline genome [12] with Megablast [41]. Isoforms containing sequence from more than one paralogous locus were identified by choosing the best hit for each germline sequence comprising the isoform, then filtering for isoforms containing segments from two or more different germline loci.

\section{RNA-Seq}

We prepared RNA-seq libraries from vegetatively-growing, starved, and encysted Oxytricha cells. The vegetative culture was grown according to the same procedure used for collecting MAC DNA. Cells for starved and cyst libraries were placed in a clean dish and incubated at $4{ }^{\circ} \mathrm{C}$ and room temperature, respectively, for 5 days. RNA for the starved and vegetative samples was extracted using TRIzol $^{\circ} \mathrm{Re}-$ agent (Life Technologies ${ }^{\mathrm{rm}}$ ). RNA for the encysted sample was extracted using $0.25 \mathrm{~mm}$ silica carbide beads in the UltraClean Microbial RNA Isolation Kit (MO Bio). Three replicates of vegetative cell RNA, three replicates of encysted cell RNA, and one replicate of $4{ }^{\circ} \mathrm{C}$-starved RNA were prepared with the Epicentre Stranded kit, along with a no-RNA input control. cDNA samples were amplified in 12 PCR cycles. Library preparation and sequencing was performed by the Lewis-Sigler Institute for Integrative Genomics Sequencing Core Facility using the Illumina Truseq Library Prep Kit.

\section{Gene prediction}

We used a gene prediction model trained on Oxytricha data and presented in [14] in conjunction with AUGUSTUS 3.3.1 [42] to predict genes for all three assemblies. We used the RNA-seq data collected from vegetatively-growing, starved, and encysted cells; previously-published RNA-seq collected from cells undergoing conjugation and genome rearrangement collected from vegetatively-growing, starved, and encysted cells; (at 0, 10, 20, 40, and $60 \mathrm{~h}$ after cells were mixed to initiate mating); and transcription start site data [10] to provide hints to the gene prediction software. We mapped reads to the genomes using HISAT2 v2.0.5 [43], 
then generated hints files according to the instructions on the AUGUSTUS web site [44]. We ran AUGUSTUS with the options --UTR = on and --alternatives-fromevidence $=$ true. We annotated the proteins using PANNZER2 [45] using default parameters and predicted protein domains using Interproscan 5 RC5 [46] using default parameters.

\section{Subsampling analysis}

We took random subsets of one, two, four, six, seven, and all eight of the flow cells from the 2015 sequencing run and used them to complete de novo Oxytricha assemblies. The reads were first filtered using the same methodology used for the primary assembly, then error corrected using either the PBcR pipeline or ECTools. For the one- and two-flow cell subsets corrected by PBcR, we used the recommended high-sensitivity parameter settings intended for low coverage assemblies $(\mathrm{QV}=52$ asmOvlErrorRate $=$ $0.1 \quad$ asmUtgErrorRate $=0.06 \quad$ asmCgwErrorRate $=0.1$ asmCnsErrorRate $=0.1$ asmOBT $=1$ asmObtErrorRate $=$ 0.08 asmObtErrorLimit $=4.5$ utgGraphErrorRate $=0.05$ utgMergeErrorRate $=0.05$ ). Otherwise, all settings used were the default. After error correction, reads were assembled using Celera assembler. To assess genome completeness, we mapped corrected reads and assembled contigs against the previously published Oxytricha assembly and counted the number of contigs at least $80 \%$ covered by either a single read or a single contig from the de novo assembly.

\section{Statistical analysis}

We carried out all statistical analyses in the R programming environment [47] and used the ggplot2 package [48] to generate figures.

\section{Abbreviations}

BUSCO: Benchmarking Universal Single-Copy Orthologs; CEG: Core Eukaryotic Genes; SMRT: Single-Molecule Real-Time

\section{Acknowledgements}

We thank Rafik Neme, V. Talya Yerlici, and other members of the Landweber lab for feedback on the genome assembly, Jingmei Wang for general laboratory assistance, and Jessica Wiggins, Wei Wang, and Donna Storton of the Princeton Sequencing Core Facility for assistance with Illumina library preparation and sequencing and Sajan Saini and Leslie Beh for feedback on the manuscript draft.

We also thank the Jonoska research group at the University of South Florida for providing public hosting of our data.

\section{Authors' contributions}

JRB isolated nuclei, extracted genomic DNA, and contributed to writing methods. RPS prepared libraries, performed sequencing, and contributed to writing methods. ERH extracted RNA, constructed libraries, and contributed to writing methods. KAL, JSP and SM carried out genome assembly, performed computational analyses, and prepared the manuscript, together with LFL. All authors have read and approved the final version of this manuscript.

\section{Authors' information}

Not applicable.

\section{Funding}

This work was supported by National Institutes of Health grants GM59708, GM109459 and GM122555 to LFL, and 1F32GM099462 to JRB, and NSF grant DMS-1800443/1764366. The funding sources had no role in this study.

\section{Availability of data and materials}

All sequencing data used in this supporting the conclusions of this article are available to the public. DNA sequencing reads used for error correction can be accessed from the Short Read Archive (http://www.ncbi.nlm.nih.gov/ sra) under accession no. SRX190400.

The complete hybrid PacBio assembly has been deposited at DDBJ/ENA/ GenBankunder the accession AMCR00000000. The version described in this paperis version AMCR02000000 (under BioProject PRJNA74629), also available at http://knot.math.usf.edu/data/external/dataMAC310/pacbio_mac_final.fa (this assembly replaces the previous short read-based assembly). The pure PacBio assembly is available at http://knot.math.usf.edu/data/external/dataMAC310/pacbio_pure_final.fa. The raw PacBio sequencing reads (SRX2335607 and SRX2335608) and vegetative (SRX2354037), starved (SRX2354036), and encysted-cell (SRX2354038) RNA-seq reads used for gene prediction are available at NCBI under BioProject PRJNA352762.

\section{Ethics approval and consent to participate}

Not applicable.

\section{Consent for publication}

Not applicable.

\section{Competing interests}

The authors declare that they have no competing interests.

\section{Author details}

${ }^{1}$ Departments of Biochemistry \& Molecular Biophysics and Biological Sciences, Columbia University, New York, NY 10032, USA. ²Lewis-Sigler Institute for Integrative Genomics, Princeton University, Princeton, NJ 08544, USA. ${ }^{3}$ Department of Biology, American University, 4400 Massachusetts Avenue, NW, Washington, DC 20016, USA. ${ }^{4}$ Icahn Institute and Department of Genetics and Genomic Sciences, Icahn School of Medicine at Mount Sinai, New York, NY 10029, USA. ${ }^{5}$ Watson School of Biological Sciences, One Bungtown Road, Cold Spring Harbor, NY 11724, USA.

Received: 11 July 2019 Accepted: 15 October 2019

Published online: 30 December 2019

\section{References}

1. National Center for Biotechnology Information. Genome Information by Organism. http://www.ncbi.nlm.nih.gov/genome/browse/. Accessed 21 Aug 2018.

2. Akpinar BA, Yuce M, Lucas S, Vrana J, Buresova V, Dolezel J, Budak H. Molecular organization and comparative analysis of chromosome 5B of the wild wheat ancestor Triticum dicoccoides. Sci Rep. 2015;5:10763.

3. Spannagl M, Martis MM, Pfeifer M, Nussbaumer T, Mayer KF. Analysing complex Triticeae genomes - concepts and strategies. Plant Methods. 2013; 9(1):35.

4. Aury JM, Jaillon O, Duret L, Noel B, Jubin C, Porcel BM, Segurens B, Daubin $\checkmark$, Anthouard V, Aiach N, et al. Global trends of whole-genome duplications revealed by the ciliate Paramecium tetraurelia. Nature. 2006:444(7116):171-8.

5. Berlin K, Koren S, Chin CS, Drake JP, Landolin JM, Phillippy AM. Assembling large genomes with single-molecule sequencing and locality-sensitive hashing. Nat Biotechnol. 2015;33(6):623-30.

6. CLC I, Loose M, Tyson JR, de Cesare M, Brown BL, Jain M, Leggett RM, Eccles DA, Zalunin V, Urban JM, et al. MinION Analysis and Reference Consortium: Phase 1 data release and analysis. F1000Res. 2015;4:1075.

7. Chaisson MJ, Huddleston J, Dennis MY, Sudmant PH, Malig M, Hormozdiari F, Antonacci F, Surti U, Sandstrom R, Boitano M, et al. Resolving the complexity of the human genome using single-molecule sequencing. Nature. 2015;517(7536):608-11.

8. Chaisson MJP, Sanders AD, Zhao X, Malhotra A, Porubsky D, Rausch T, Gardner EJ, Rodriguez OL, Guo L, Collins RL, et al. Multi-platform discovery of haplotype-resolved structural variation in human genomes. Nat Commun. 2019;10(1):1784. 
9. Koren S, Phillippy AM. One chromosome, one contig: complete microbial genomes from long-read sequencing and assembly. Curr Opin Microbiol. 2015;23:110-20.

10. Beh LY, Debelouchina GT, Clay DM, Thompson RE, Lindblad KA, Hutton ER, Bracht JR, Sebra RP, Muir TW, Landweber LF. Identification of a DNA N6adenine methyltransferase complex and its impact on chromatin organization. Cell. 2019;177(7):1781-96 e1725.

11. Gottschling DE, Zakian VA. Telomere proteins: specific recognition and protection of the natural termini of Oxytricha macronuclear DNA. Cell. 1986; 47(2):195-205.

12. Chen X, Bracht JR, Goldman AD, Dolzhenko E, Clay DM, Swart EC, Perlman $\mathrm{DH}$, Doak TG, Stuart A, Amemiya CT, et al. The architecture of a scrambled genome reveals massive levels of genomic rearrangement during development. Cell. 2014;158(5):1187-98.

13. Prescott DM. The DNA of ciliated protozoa. Microbiol Rev. 1994;58(2):233-67.

14. Swart EC, Bracht JR, Magrini V, Minx P, Chen X, Zhou Y, Khurana JS, Goldman AD, Nowacki M, Schotanus K, et al. The Oxytricha trifallax macronuclear genome: a complex eukaryotic genome with 16,000 tiny chromosomes. PLoS Biol. 2013;11(1):e1001473.

15. Braun J, Nabergall L, Neme R, Landweber LF, Saito M, Jonoska N. Russian Doll Genes and Complex Chromosome Rearrangements in Oxytricha trifallax. G3 (Bethesda). 2018;8(5):1669-74.

16. Burns J, Kukushkin D, Chen X, Landweber LF, Saito M, Jonoska N. Recurring patterns among scrambled genes in the encrypted genome of the ciliate Oxytricha trifallax. J Theor Biol. 2016;410:171-80.

17. Yerlici VT, Landweber LF. Programmed Genome Rearrangements in the Ciliate Oxytricha. Microbio/ Spectr. 2014;2(6).

18. Quail MA, Smith M, Coupland P, Otto TD, Harris SR, Connor TR, Bertoni A, Swerdlow HP, Gu Y. A tale of three next generation sequencing platforms: comparison of ion torrent, Pacific Biosciences and Illumina MiSeq sequencers. BMC Genomics. 2012;13:341.

19. Laehnemann D, Borkhardt A, McHardy AC. Denoising DNA deep sequencing data-high-throughput sequencing errors and their correction. Brief Bioinform. 2016;17(1):154-79.

20. Simao FA, Waterhouse RM, loannidis P, Kriventseva EV, Zdobnov EM. BUSCO: assessing genome assembly and annotation completeness with single-copy orthologs. Bioinformatics. 2015;31(19):3210-2.

21. Waterhouse RM, Seppey M, Simao FA, Manni M, loannidis P, Klioutchnikov G, Kriventseva EV, Zdobnov EM. BUSCO applications from quality assessments to gene prediction and phylogenomics. Mol Biol Evol. 2017; 35(3):543-548.

22. Chen X, Jung S, Beh LY, Eddy SR, Landweber LF. Combinatorial DNA rearrangement facilitates the origin of new genes in ciliates. Genome Biol Evol. 2015;7(10):2859-70.

23. Chen X, Jiang Y, Gao F, Zheng W, Krock TJ, Stover NA, Lu C, Katz LA, Song W. Genome analysis of the unicellular eukaryote Euplotes vannus reveals molecular basis for sex determination and tolerance to environmental stresses. bioRxiv. 2018:357715

24. Herrick G, Cartinhour SW, Williams KR, Kotter KP. Multiple sequence versions of the Oxytricha fallax 81-MAC alternate processing family. J Protozool. 1987; 34(4):429-34.

25. Klobutcher LA, Huff ME, Gonye GE. Alternative use of chromosome fragmentation sites in the ciliated protozoan Oxytricha nova. Nucleic Acids Res. 1988;16(1):251-64.

26. Bracht JR, Wang X, Shetty K, Chen X, Uttarotai GJ, Callihan EC, McCloud SS, Clay DM, Wang J, Nowacki M, et al. Chromosome fusions triggered by noncoding RNA. RNA Biol. 2017;14(5):620-31.

27. Fang W, Wang X, Bracht JR, Nowacki M, Landweber LF. Piwi-interacting RNAs protect DNA against loss during Oxytricha genome rearrangement. Cell. 2012;151(6):1243-55

28. Neeb ZT, Hogan DJ, Katzman S, Zahler AM. Preferential expression of scores of functionally and evolutionarily diverse DNA and RNA-binding proteins during Oxytricha trifallax macronuclear development. PLoS One. 2017;12(2): e0170870.

29. An D, Cao HX, Li C, Humbeck K, Wang W. Isoform Sequencing and State-ofArt Applications for Unravelling Complexity of Plant Transcriptomes. Genes (Basel). 2018:9(1).

30. Pootakham W, Sonthirod C, Naktang C, Ruang-Areerate P, Yoocha T, Sangsrakru D, Theerawattanasuk K, Rattanawong R, Lekawipat N, Tangphatsornruang $\mathrm{S}$. De novo hybrid assembly of the rubber tree genome reveals evidence of paleotetraploidy in Hevea species. Sci Rep. 2017;7:41457.
31. Rhoads A, Au KF. PacBio sequencing and its applications. Genomics Proteomics Bioinformatics. 2015;13(5):278-89.

32. Werner MS, Sieriebriennikov B, Prabh N, Loschko T, Lanz C, Sommer RJ. Young genes have distinct gene structure, epigenetic profiles, and transcriptional regulation. Genome Res. 2018;28(11):1675-87.

33. Pacific BioSciences. SMRT Pipe Reference Guide v2.1. https://github.com/ PacificBiosciences/SMRT-Analysis/wiki/SMRT-Pipe-Reference-Guide-v2.1. Accessed 6 Jun 2016.

34. Gurtowski J. Error correction and assembly complexity of single molecule sequencing reads. https://github.com/jgurtowski/ectools. Accessed 6 Jun 2016.

35. Lee H, Gurtowski J, Yoo S, Marcus S, McCombie WR, Schatz M. Error correction and assembly complexity of single molecule sequencing reads. bioRxiv. 2014;006395

36. Rognes TM F, Flouri T, Quince C, Nichols B, VSEARCH. https://github.com/ torognes/vsearch. Accessed 6 Jun 2016.

37. BBTools - DOE Joint Genome Institute. http://jgi.doe.gov/data-and-tools/ bbtools/. Accessed 3 Jul 2017.

38. Walker BJ, Abeel T, Shea T, Priest M, Abouelliel A, Sakthikumar S, Cuomo CA, Zeng Q, Wortman J, Young SK, et al. Pilon: an integrated tool for comprehensive microbial variant detection and genome assembly improvement. PLoS One. 2014;9(11):e112963.

39. Li H: Aligning sequence reads, clone sequences and assembly contigs with BWA-MEM. 2013; Preprint at https://arxiv.org/abs/1303.3997.

40. Quinlan AR, Hall IM. BEDTools: a flexible suite of utilities for comparing genomic features. Bioinformatics. 2010;26(6):841-2.

41. Camacho C, Coulouris G, Avagyan V, Ma N, Papadopoulos J, Bealer K, Madden TL. BLAST+: architecture and applications. BMC Bioinformatics. 2009; 10:421.

42. Stanke M, Morgenstern B: AUGUSTUS: a web server for gene prediction in eukaryotes that allows user-defined constraints. Nucleic Acids Res. 2005; 33(Web Server issue):W465-W467.

43. Kim D, Langmead B, Salzberg SL. HISAT: a fast spliced aligner with low memory requirements. Nat Methods. 2015;12(4):357-60.

44. Incorporating RNA-Seq into AUGUSTUS [Internet]. [cited 2016 Jun 5]. Available from: http://augustus.gobics.de/binaries/readme.rnaseg.html

45. Toronen P, Medlar A, Holm L. PANNZER2: a rapid functional annotation web server. Nucleic Acids Res. 2018;46(W1):W84-8.

46. Jones $P$, Binns D, Chang HY, Fraser M, Li W, McAnulla C, McWilliam H, Maslen J, Mitchell A, Nuka G, et al. InterProScan 5: genome-scale protein function classification. Bioinformatics. 2014;30(9):1236-40.

47. R Core Team. R: A language and environment for statistical computing. $R$ Foundation for Statistical Computing, Vienna, Austria. 2017. www.R-project.org

48. Wickham H. ggplot2: Elegant Graphics for Data Analysis. New York: Springer-Verlag; 2016.

\section{Publisher's Note}

Springer Nature remains neutral with regard to jurisdictional claims in published maps and institutional affiliations.
Ready to submit your research? Choose BMC and benefit from:

- fast, convenient online submission

- thorough peer review by experienced researchers in your field

- rapid publication on acceptance

- support for research data, including large and complex data types

- gold Open Access which fosters wider collaboration and increased citations

- maximum visibility for your research: over $100 \mathrm{M}$ website views per year

At BMC, research is always in progress.

Learn more biomedcentral.com/submissions 